\title{
La Enseñanza del Derecho como un Espacio de Conflicto en una Sociedad Diversa: Aportes para el Debate
}

\author{
Teaching Law as a Space of Conflict in a Diverse Society: \\ Contributions to Debate
}

Dr. Efrén Ernesto Guerrero Salgado, PhD.

Docente Titular Principal PUCE Quito

Artículo Original (Investigación)

RFJ, No. 3, 2018, pp. 81-97, ISSN 2588-0837

RESUMEN: el presente texto busca hacer aportar con elementos de análisis respecto del papel de las escuelas de Derecho de nuestro país como espacios de construcción y reproducción de formas de poder que podrían conspirar, mediante sus contenidos y prácticas, con un sistema de cambio social. La hipótesis que maneja este trabajo es que, al analizar las prácticas como Facultades, y los mecanismos sociales que se generan en las prácticas jurídicas, lo que se hace es tener un sistema jurídico formal y funcional pero que en el sentido práctico perpetua los errores y vicios que al menos en teoría, los docentes de derecho suponen luchar, todo esto en medio de un conflicto entre continuidad de cambio, signado entre el positivismo más tradicional, y escuelas críticas.

PALABRAS CLAVES: Ecuador, poder, Derecho, Educación Superior, andragogía.

ABSTRACT: the present text seeks to contribute with elements of analysis regarding the role of the law schools of Ecuador as spaces of construction and reproduction of forms of power that could conspire, through its contents and practices, with a system of social changes. The hypothesis that handles this work is that, when analyzing the practices like Faculties, and the social mechanisms that are generated in the legal practices, what is done is to have a formal and functional legal system but in the practical sense perpetuates the errors and Vices that at least in theory, teachers of law supposed to fight, all this in the middle of a conflict between continuity of change, signified between the more traditional positivism, and critical schools. 
KEY WORDS: Ecuador, power, Law, Higher Education, andragogy.

\section{INTRODUCCIÓN}

Quien suscribe estas líneas ha tenido el honor de ser durante una decena de años docente de Derecho. Creo que ha sido, sin lugar a dudas, le mejor decisión de mi vida, ya que me ha servido especializarme en el Derecho como un objeto de estudio y forma de investigación. Para eso, creo -en la medida que la ciencia implica creer que sus mecanismos son funcionales- que las normas son las herramientas más importantes para los cambios sociales, ya que las grandes luchas de los pueblos empiezan y terminan o en la expedición de una norma con carácter de obligatorio, o en la puerta de un juzgado.

Pero, para que eso suceda, es necesaria la construcción de la norma jurídica capaz de ser sostenida por un grupo de ideas lo suficientemente fuerte para que se vuelvan teorías lo suficientemente potentes como para que expliquen la experiencia social. Ese es el papel del Derecho como elemento educativo: se toma a personas con diferentes maneras de ver la vida, la moral y lo más importante, lo "justo" y "lo injusto", y se les enseña la mejor manera posible de aplicar e interpretar el Derecho. Ese último punto es el que debería causarnos menor conflicto, ya que enseñar Derecho no solo debe parecer justo, sino debe serlo. Debe ayudar a la construcción de una sociedad donde el ejercicio de las libertades se dé en su mejor contenido, y que al mismo tiempo genere respetabilidad capital social y reconocimiento.

Muy pocas carreras o facultades presumen tanto de ser "esposas del César", como las jurídicas. En un mundo plural, y secularizado al extremo, los espacios de formación jurídica siguen mostrándose como un espacio de extremismos, y de disputas graves entre maneras de ver el mundo. Ese ejemplo puede verse respecto a una disertación realizada en una universidad ecuatoriana a finales del año 2016 respecto al tema de femicidio: el autor afirmaba que el tipo penal era producto de una construcción filosófica contraria a los intereses de un sector social, y que era, en suma, un producto de un lobby social peligroso. Al respecto, cabe preguntarse, independientemente de los méritos académicos de esa disertación, la siguiente pregunta teórica: es posible construir una educación jurídica capaz de mejorar el Derecho, o los errores de la práctica profesional son presentados en el mundo jurídico como un espacio de mejora y gestión de la realidad. 
Esa pregunta se relaciona con: a) la forma en que se enseñan las instituciones jurídicas; b) un contexto social de rechazo a ciertas instituciones de apertura de contenidos de derechos, tanto por quienes detentan el poder político, quienes quisieran obtenerlo; cosa que responde a nuestra crisis mundial del sistema democrático; c) un mínimo moral histórico respecto a nuestras capacidad de confiar en los demás y en gestión del sistema de mantenimiento de la sociedad, y d) una falla estructural del valor del conocimiento, que en el plano jurídico Ferrajoli llama "rechazo civil a la Constitución" (Ferrajoli, 2013) y en el plano del cine se ha llamado Idiocracia: una combinación letal nihilismo, inmediatismo, liquidez del conocimiento y falta de pluralismo que pueden llevar al desencadenamiento de derivas autoritarias, tanto a nivel jurídico como social (Tremblay, 2016).

Con esos antecedentes, las Facultades de Derecho ecuatorianas se encuentran en una disyuntiva, probablemente la más importante de su historia: la necesidad de una construcción de un Derecho que asegure -valga la redundancia- del Estado de Derecho, y que, al mismo tiempo, sirva como un medio para la construcción de una sociedad abierta. El presente texto busca hacer aportar con elementos de análisis de esta disyuntiva, al respecto del papel de las escuelas de Derecho de nuestro país como espacios de construcción y reproducción de formas de poder que podrían conspirar, mediante sus contenidos y prácticas, con un sistema poliárquico de gran escala. La hipótesis que maneja este trabajo es que, al analizar las prácticas como Facultades, y los mecanismos sociales que se generan en las prácticas jurídicas, lo que se hace es tener un sistema jurídico formal y funcional pero que en el sentido práctico perpetua los errores y vicios que al menos en teoría, los docentes de Derecho suponen luchar, todo esto en medio de un conflicto entre continuidad de cambio, signado entre el positivismo más tradicional, y escuelas críticas.

Para esto, dividiremos este texto en dos: en primer lugar se hablará de la Universidad y las Facultades de Jurisprudencia como espacios de construcción de poder. Se analizará como en el momento actual, hay una confluencia importante de un choque entre órdenes de reproducción de poder y cuáles son sus consecuencias. Las dos últimas secciones proponen hacer un estudio prospectivo. Se enfocará en los errores que como espacios de formación se hacen hacia los adultos, y finalmente comprender de forma propositiva la existencia del conflicto, y cómo podemos utilizarlo de manera creativa a través de las ideas utópicas, para construir un mejor Derecho y como consecuencia, una mejor sociedad. 


\section{EL EJERCICIO DEL DERECHO COMO UN MECANISMO DE EJERCICIO DE PODER}

La Ciencia Jurídica es un mecanismo de mantenimiento de un determinado status quo. Esa es una verdad de Perogrullo que no puede ser obviada en el proceso de enseñanza y aprendizaje del Derecho. Considerando que el uso y abuso de las normas se manifiestan en mecanismos de imperium del Estado, es lógico que el uso de ese poder no es aséptico, sino que tiene influencias ideológicas y políticas. Este tema no se manifiesta ni en la literatura ni la práctica diaria ecuatoriana; al parecer existe una cierta cultura de urbanidad dentro del claustro universitario, que puede manifestarse por un fenómeno de "puerta giratoria": los y las docentes tienen una relación entre el sector público y el sector privado de entrada y salida (y convivencia), que vuelve al debate sobre la vida universitaria y sus consecuencias en el mundo jurídico como marginal y poco necesario.

En contraposición, la actuación de los docentes universitarios en las Facultades, y el claustro universitario como una herramienta de gestión de poder, es un tema ampliamente relatado por la doctrina. Para poder resumir el estado del arte, lo que se hará es analizar lo que plantean los textos frente al tema:

a. Función Social de la Universidad (y la carrera de Derecho): en un país con profundas desigualdades sociales, económicas y raciales, las universidades se han vuelto fuertes mecanismo de ascenso social: la abogacía es una profesión que da un capital social, simbólico, y especialmente jurídico, ya que da a sus operadores las herramientas necesarias para poder modular las acciones de los ciudadanos. Eso implica, como la gran mayoría de prerrogativas individuales, una función individual, signada en la posibilidad de los ciudadanos, y una función social, basada en cómo el uso de las normas su aprendizaje se traduce en una sociedad mejor. Eso, implica que lo aprendido en la Facultad se traduzca en una mejor capacidad de gestión y uso del ordenamiento jurídico. Esa no es una preocupación nueva. De hecho sucedía en Francia hace más de un siglo:

"Hay que decir que la humilde consideración que se da hoy en nuestros tribunales a lo que antes era ciencia jurídica; la manía de juzgar y suplicar solo los hechos; la creciente elasticidad de los textos y la perezosa indisciplina de la interpretación; la sustitución de la equidad, es decir, la arbitrariedad, el imperio de la ley, han 
creado tendencias perjudiciales que amenazan todos los buenos hábitos, los nobles escrúpulos y todas las tradiciones quizás estrictas pero saludables de los tiempos pasados “ (Ferry, 1868: 3).

En ese orden de cosas, la eterna discusión de quienes enseñamos y aplicamos el Derecho, es lograr trasladar no solo las enseñanzas sino los valores respecto a la realidad. De otra manera no se construye educación, sino el mero uso de herramientas, desprovistas de una justificación sostenida en los más altos ideales humanos. Al respecto, la primera discusión que debe hacerse en las universidades es si las actividades de sus alumnos docentes mejora o disminuye el ejercicio de los derechos ciudadanos existentes en el Ecuador.

El ejercicio en el caso ecuatoriano, fue la construcción de una obligación legal, basada en la norma contenida en el Código Orgánico de la Función Judicial, en forma de prácticas profesionales. ¿Es suficiente eso como el aporte de la enseñanza del Derecho a la Sociedad? Frente a eso, hay que hacer una distinción entre el aporte visible, relacionado con lo que se hace en el mundo físico; y el aporte invisible, que se sostiene en la manera de ser abogado, que es lo que la Facultad de Derecho entrega a la sociedad.

En ese orden de cosas, las Facultades de Derecho pueden volverse espacios de reproducción de mecanismos de poder, o de cambio del sistema social. Dentro de sus paredes y sus aulas, las actividades jurídicas y de enseñanza son un permanente debate a favor y en contra de la realidad. En tal razón, cada universidad debe ser clara respecto a lo que quiere entregar a la sociedad en términos de debate. Al respecto, una heurística de actuación puede ser lo que se ve en la historia, entre la resistencia activa a la reforma administrativa hecha por las escuelas de derecho francesas del siglo XIX y XX (Savage, 2008), o el apoyo desde la academia americana al movimiento de derechos civiles (Shaffer \& Shaffer, 1960). Dentro de esos dos extremos, entre continuidad y cambio, cada Facultad debe ubicarse, para poder reproducir poder de una manera acorde con su interés institucional y su sentido histórico.

b. Temas de enseñanza: los pensum de la gran mayoría de Facultades, amén del amplio proceso de reglamentación de las carreras de interés público realizada desde las agencias de control estatales, se basa efectivamente en temas de interés privado, signados todavía en el Código Civil. ¿Es esa la única forma correcta de hacerlo? En 
relación con eso, lo que se puede apreciar es que nuestro paradigma románico-occidental ha generado una cierta escolaridad en los temas de estudio y los constriñen a temas cerrados, y planes de estudios limitados a cierto número de horas. En ese escenario cada Facultad de Derecho debería en este escenario encontrar una manera de demostrar una ventaja competitiva frente a otras. Eso no sucede en el caso ecuatoriano, ya que las instituciones de Educación Superior, en vez de enfocarse construir una identidad, se ven limitadas por presiones administrativas técnicas. Esa es una muestra de que Bourdieu confirme su teoría: la existencia de actores con mayo capital simbólico y político (El Estado y sus agencias) frente a actores cuyo mayor capital es simbólico. Para las instituciones de educación superior ecuatorianas, el reto es evocar el poder de lo simbólico, y recrear los mecanismos simbólicos (reputación, legado, estima social), frente a la regulación y el poder económico.

c. Contenidos de la enseñanza: sobre ese tema bien vale hacer una crítica estructural a toda la enseñanza ecuatoriana. La gran parte de la manera en que enseñamos y aprendimos Derecho se ha basado en el sistema kelseniano. Al respecto, Kelsen y otros autores considerados clásicos, son entendidos a través de fuentes secundarias, y desprovistos de su papel político, su sentido histórico y sus alcances dentro de una realidad determinada. En este sentido, es conveniente detenerse en la figura de Kelsen. Hay que tener presente que los textos del autor vienés, lejos de ser un análisis puramente técnico, tiene un interés político: separar al derecho de los contenidos no propios del mismo, y que no se vuelva una ciencia política. Eso supone que el Derecho recoja elementos de las ciencias exactas y que eso se manifiesta en formas jurídicas exactas. Al respecto el mismo Kelsen afirmó lo siguiente:

Es una ilusión suponer que una teoría de la ley natural podría dar una respuesta absoluta a la pregunta sobre la base de la validez de la ley positiva. Tal teoría ve la base de la validez del Derecho positivo en la ley natural, es decir, en un orden establecido por la naturaleza como autoridad suprema, por encima de la autoridad del legislador (Kelsen, 1992: 7).

Eso muestra que la acción social y política se inscribe en el sistema público, y educativo. Eso ha sido visto fuertemente en caso latinoamericano, que ha utilizado el sistema de Kelsen como un medio para hacer patente valores, sin tener en cuenta que por ser 
una Teoría Pura, por basarse en un sistema de gestión y no de interpretación del Derecho per se (Medina, 2004).

Como se puede ver, ni el mismo autor estaba asumiendo su teoría como la más adecuada o la mejor aplicable. Se la ha propuesto como la que didácticamente resuelve mejor los problemas básicos de la importancia jurídica y la que técnicamente completa los vacíos existentes en la ciencia. Pero como toda teoría solamente explica el mundo de una manera, sin ser un reflejo de la realidad. Lo que nos ha sucedido a los abogados es que hemos explicado el Derecho a través de la repetición simple de los postulados de una (de tantas) explicaciones de la realidad. Eso por sí solo, es reproducir el poder cultural, y transformarlo en cambios en el campo social. Es por esto que las Facultades de Derecho deben tener en cuenta lo importante de las teorías que se enseñan, y como estas se trasladan en productos en la realidad.

d. Sobre los mecanismos de enrolamiento de docentes: Al respecto, la literatura establece tres elementos de discusión básicos:

El primero, y quizás el más obvio, es el atractivo económico de la enseñanza del Derecho como profesión. La segunda es la naturaleza de la profesión de la enseñanza de Derecho en sí: ¿Cuál es la persona que busca enseñar el Derecho como parte de su vida profesional? La tercera pregunta se refiere al sentimiento a largo plazo de logro y satisfacción en la carrera. (...). Una vez que hemos examinado estas preguntas tal vez podamos entonces abordar una cuarta pregunta: ¿Qué se puede hacer para que la enseñanza del Derecho sea una profesión más atractiva? (Christie, 1987: 306).

En el caso ecuatoriano se ha generado este espacio como un mecanismo de reproducción de poder básico. En primer lugar, y hasta años recientes, enseñar Derecho a tiempo completo era un camino a una vida económica bastante modesta, en comparación de los alicientes que suceden en el mundo privado o en el sector público ecuatoriano. Un aumento de sueldos ha vuelto a la profesión adecuada para que una persona pueda generar un proyecto serio de vida en relación con otros espacios. Sin embargo, un reto a mejorar en la instituciones de Educación Superior es mejorar y potenciar la planta de docentes de tiempo completo y tiempos parcial, de forma que el capital social y cultural de su staff pueda compararse con aquel del sector privado. Hay que asumir que la educación universitaria no solamente es un tema de vocación, ya que ese es un justificativo que impide crear una 
cultura de calidad total, en la que la formación integral de la persona es una prioridad. Asimismo, implica hacer que una obsesión por los títulos, producto de una adecuación tecnocrática por parte de las agencias estatales (comprensible y entendible en la forma), genere un espacio con un equilibrio adecuado con la experiencia de la academia con la experiencia del ejercicio de la profesión. Se debe hacer que el académico de Derecho sea una profesión dentro de la Ciencia Jurídica y no un espacio mínimo y aislado.

La segunda pregunta es relativa al profesional del Derecho como persona. En ese aspecto, la violencia en el aula, es el peor mecanismo de repetición de poder: la existencia de historias y anécdotas peligrosamente y desgraciadamente jocosas de racismo, clasismo, o misoginia por parte de docentes universitarios, pueden entenderse como un mecanismo de reproducción de un capital simbólico y cultural que poco aporta en la creación de valores democráticos. En ese sentido, las relaciones entre estudiantes y profesores en el marco de la actividad académica que desarrolla nuestra Facultad, como toda relación entre adultos, deben fundarse en relaciones basadas en el mutuo respeto y en el reconocimiento de la dignidad que todos tenemos como seres humanos.

Por tal razón, en el caso de las y los estudiantes, su situación de razón de ser de toda la actividad educativa motiva a que se les brinde una protección especial. Debe tenerse en cuenta que los estudiantes se encuentran en una situación de asimetría de poder que obliga a las autoridades a generar mecanismos de protección. De no hacerse de esta manera, se impide la igualdad de oportunidades, la seguridad personal, la integridad física, el libre desarrollo de la personalidad, la igualdad ante la ley, la no injerencia arbitraria en la vida privada y la protección frente a ataques a su honra o reputación o a condiciones de empleo justas y favorables. Para evitar daños personales, grupales e institucionales, las Facultades no solo deben buscar escoger buenos abogados, sino también buenas personas. La gestión del talento humano debe buscar la construcción de personas integrales, que puedan ayudar a la construcción de un Derecho capaz de superar la teoría y poder llegar a actuar desde la empatía y la construcción de la igualdad completa entre seres humanos.

La tercera pregunta, es construir una gestión de la satisfacción de la carrera académica como un espacio de la actividad universitaria. Este hecho no solo habla de satisfacción académica, sino de calidad 
de vida social. Fuera de lo que se supone desde fuera del mundo universitario, el trabajo dentro delas Facultades es poco visible, altamente competitivo, con pocos alicientes, egos en conflicto y con fuertes espacios de presión. La solución a ese tema debería ser la humildad máxima, especialmente frente al mundo jurídico. Una de los hechos, en un mundo basado en los títulos, y dentro de ellos, de dónde y cómo se ha obtenido, es que se realizan maestrías o doctorados como un mecanismo para construir una imagen en el sistema social y proteger su versión del status quo. Pero eso no sirve de nada ahora. Necesitamos doctores con hambre de gloria, y de arriesgar mucho por cambios estructurales en el sistema académico. Pero para eso necesitamos humildad, para construir un espacio de colaboración y sinergias entre las ciencias (Melin \& Janson, 2006) ${ }^{35}$. Además, la salud emocional y mental de los docentes debe ser discutida y es un tema que debe analizarse con franqueza en el espacio académico. Las características de los académicos y las académicas se entrelazan para producir un sistema ecológico que, paradójicamente, puede generar espacios de construcción de conocimiento, pero también circunstancias personales de "baja supervisión, baja visibilidad del desempeño, libre de demandas de tiempo, y estándares de desempeño vagamente definidos y no forzados de rendimiento" (Thorseson, 1984:56), que puede llevar a episodios poco visibilizados de depresión, ansiedad y disminución del trabajo académico (University of California, Berkeley, 2014).

Como corolario de esta reflexión, es necesario construir una universidad atractiva a aquellos que quieran hacer carrera universitaria, con buenos, sueldos, espacios de trabajo adecuado a su profesión, cargas de trabajo adecuadas, y la posibilidad de ejercer su trabajo con un libertad académica equilibrada con estándares académicos de excelencia, que no sean solamente un discurso publicitario, sino un ejercicio universitario basado en su idea fundacional en el medievo: un lugar donde acumular y construir con la mejor gente posible, la mejor ciencia posible.

e. Finalmente, sobre el de la ética profesional dentro del pensum universitario. Sobre este tema, y considerando el poder fáctico las universidades como reproductoras de poder, no hay otro espacio que

35 A nivel personal, mi tránsito entre el derecho hacia las ciencias políticas me ha demostrado lo limitada y débil que es la ciencia jurídica para explicarlos hechos sociales y la necesidad de que se incluyan inputs de la ciencia política en su funcionamiento. 
se construya una formación deontológica clara tanto en las prácticas como en los productos académicos. Eso implica una relación entre los actores dentro y fuera de la universidad en una relación de doble vía basada en que los profesionales de Derecho no pueden enseñar el deber ser de la norma, y fuera de ella, torcer la normatividad. ${ }^{36} \mathrm{Al}$ respecto, hay son responsables claros dentro de la academia y el mundo profesional:

La codicia no es buena. La avaricia de los abogados y profesores de Derecho es un ejemplo. A medida que las vidas de los abogados superiores -especialmente los de las empresas de élite- se dominan por la persecución de horas facturables y como las vidas de los profesores de Derecho -especialmente las de las escuelas de élite- se ven dominadas por la búsqueda de becas y publicaciones. Los abogados y los profesores de Derecho están dando la espalda a mucho más que es importante. Y entre las más críticas de las responsabilidades que los abogados y profesores de Derecho que abandonan es la formación moral de los jóvenes - la formación de los estudiantes de Derecho y los nuevos abogados en profesionales con ética (Schiltz, 1998: 747).

Sobre esto, la reproducción de un capital simbólico que proteja, acepte y crea como "la letra colorada" (el espacio donde hay capacidad de actuar según los propios desee) de la Ley, ${ }^{37}$ es absolutamente incompatible con la profesión y la acción del Derecho. Al respecto, la doctrina propone una respuesta clara de guía moral: "Haz a los demás como quisieras que te hicieran a ti. Se honesto. Sé justo. Sé cortés. Sé compasivo. Sea fiel a su palabra. Como reconoció Abraham Lincoln, "la virtud en un abogado no es muy diferente de la decencia común en ningún otro llamado" (Schiltz, 1998: 714). De no hacerlo, el resultado probable sería lo que la academia americana llama el "declinamiento moral de la profesión”, que está signada por los siguientes elementos: La ética de los abogados está disminuyendo, tanto en el cumplimiento de los códigos éticos y en el compromiso con el bien público. En se-

36 Hay pocas frases que me molestan más como una que escuche en mi época de estudiante: "a la ley no se la viola, se la enamora" que al parecer fue pronunciada por un docente. Confío que ese esperpento sea apócrifo, pero refleja ese tipo de comportamiento de doble rasero moral que es incompatible con la enseñanza del Derecho.

$37 \mathrm{Al}$ respecto, la idea de "letra colorada" puede extraerse de los glosadores los Códigos Españoles del siglo XV. En algunos textos se plantea que existe una glosa de color rojo (colorado) que es distinta de las demás. (Rivadeneyra, 1847: 437) 
gundo lugar la profesión ha sido incapaz de controlarse adecuadamente porque su sistema disciplinario es ineficaz. En tercer lugar, sea cual fuere el mérito y la fe en el profesionalismo y en la educación jurídica, tal mérito y fe ya no ofrecen un apoyo creíble a la posición académica de que la enseñanza de la ética legal no es esencial. Por tal razón, la creciente evidencia de la conducta antiética del abogado continúa provocando demandas de una mejor enseñanza ética de las escuelas de Derecho (Pearce, 1998).

En resumen, la construcción del poder jurídico en el caso ecuatoriano, se basa en que el sistema reproduce ciertas prácticas simbólicas, culturales y técnicas que no han sido visibilizadas en la práctica diaria, y de haber sido realizadas, solamente han sido entendidas como mecanismos puramente académicos. En tal razón, la gestión del Derecho y la mejora de todos los mecanismos que construyen la educación del mismo tienen que asumir estos espacios como necesidades de debate y mejora.

\section{UNA PROPUESTA DESDE LA UTOPÍA}

En este escenario, hay que proponer una manera de enseñar Derecho para los ecuatorianos, desde la realidad del país, y con la técnica de los docentes ecuatorianos, que sea capaz de adaptar tradición y modernidad, los cambios normativos, y que pueda formar y atender a los jóvenes que vienen de la sociedad más diversa que el Ecuador ha tenido nunca. Eso no es nada fácil. De hecho, la circunstancia que vivimos en las Facultades de Derecho no es una reforma del sistema: es una singularidad social: un espacio en que las capacidades tradicionales de la Educación Universitaria deben superar el sistema regulador o los paradigmas tradicionales, con el fin de que las actividades personales afecten de forma impredecible el sistema completo (Yudkowsky, 2007). Para poder hacer que esto suceda, hay que arriesgar como individuos y grupos y construir una metodología de la construcción de la utopía. Del Derecho que queremos y de la Facultad de Jurisprudencia que queremos. Porque es realizable y necesario, para construir un ordenamiento jurídico mejor.

En primer lugar, debe suponerse el fondo del Derecho no como un mecanismo de mantenimiento del poder o de un cierto estado de situación social, sino como un ejercicio colectivo en el que no solo se busca regular lo que es, sino aquello que debería hacerse. Eso es 
consecuencia directa de la herramienta que se maneja en la Ciencia Jurídica: las normas legales, especialmente una Constitución como la ecuatoriana, con un amplio sistema de garantía de Derechos, delimita el tipo de sociedad en que vive y quiere vivir la gente (Almog, 2003: 148). Al enseñar un mecanismo de aplicar y entender el derecho, se está creando una forma de actuar y relacionarse con la gente, y se debe prever sus efectos positivos o negativos. Por tal razón, las Facultades de Derecho deben aplicar de forma expansiva un espíritu de mostrar al poder político lo que debe ser la sociedad y lo que debe ser el Derecho, de forma transparente y evitando una corrección política que puede ser peligrosa.

En segundo lugar, se debe asumir la realidad. Si deja(mos) la corrección política, el sistema jurídico es consecuencia de la falta de contenido ético de la profesión jurídica, su tendencia a la memorización y la falta de discusión de instituciones jurídicas que son entendidas de forma dogmático ${ }^{38}$. Una educación jurídica debe mostrar el Derecho como una herramienta, pero debe centrarse en la educación a favor de estudiantes competentes moralmente, pero también técnicamente competentes. No se debe olvidar una idea que:

Kelsen y Hart compartían: la ley solo será necesaria para los seres humanos vulnerables, que no tienen suficientes bienes para distribuir y cuyo comportamiento no está garantizado debido al limitado altruismo (Avilés, 2003). Por eso, es necesario que se construya una educación signada por la protección de las personas, especialmente aquellas más vulnerables, y que valore las diferencias (Ferrajoli, 1999: 81), inclusive dentro de las Facultades, que tienen una tendencia a la uniformidad que debe evitarse.

38 Los sistemas jurídicos en Latinoamérica, no actualizados a las necesidades actuales y a los avances tecnológicos, crean, en última instancia "una gran maquinaria de producir expedientes, certificaciones, notificaciones, etc., donde el juez es una suerte de administrador de tal oficina burocrática e ineficiente antes que un verdadero juez." (Binder, 2002), que lo aísla del mundo exterior, complica las posibilidad de acción y aumentan la posibilidad de recibir influencias externas que lo hagan caer en corrupción. Igualmente, y dada la gran cantidad de procesos, se ha disminuido la calidad de la argumentación jurídica: "la argumentación no tiene tanta importancia cuando lo que domina es el método memorístico... simplemente para aplicarlo (el ordenamiento) en los términos en que esté establecido por el legislador..." (Simon y Navas, 2003) 
En tercer lugar, hay que asumir responsabilidades. Las Facultades de Derecho deben asumir que el modelo de educación jurídica actual es responsable de los niveles del Estado de Derecho en el Ecuador. Eso obliga a asumir las consecuencias a nivel ético de los modelos de educación existente, por lo que es necesario que "la academia debe aceptar que una de sus funciones es preparar a los estudiantes para practicar la ley - y para practicar el Derecho éticamente. Los profesores deben "aceptar la idea de que están formando a los abogados y que entienden que el objetivo de su enseñanza en el aula es el cultivo de las cualidades que un abogado necesita para tener éxito en la práctica (Schiltz, 1998: 747). Implica entender que la fuerza del Derecho es la fijación de los límites de la acción y que el escenario de democracia delegativa (O’Donell, 2010), es imposible de apoyar desde cualquier situación.

Finalmente, hay que saber a dónde vamos. La utopía exige un lugar a donde llegar. Es un punto omega de cualquier acción de cambio, sea este estructural o una reforma administrativa básica. (Pollit \& Bouckaert, 2010), que depende de la acción de sus élites, todo proceso estructural en el que se han integrado las estructuras de poder político-económico han sido una serie de acciones en el que en determinados momentos, se ha hecho una reorganización a gran escala de sectores de la actividad del Estado (Chase, 2002). Eso debe discutirse institucional y socialmente, y centrado en el modelo de Estado y Sociedad que se busca encontrar de este proceso. ¿Cuál es el modelo de democracia que se propone desde las Facultades de Derecho? Esa pregunta es necesaria, ya que el respeto del Estado de Derecho y la democracia, ya que la actuación de cualquier instancia está encaminada no solo a la buena marcha de su propia administración sino a la defensa de las libertades individuales (Camps, 1998).

\section{CONCLUSIONES}

En conclusión, necesitamos una educación que reproduzca un nuevo tipo de poder. Una educación jurídica diversa para una sociedad diversa, capaz de entender los cambios sociales, y que genere profesionales que vean el poder profundo de la ciencia jurídica para transformar la realidad, así como el peligro ético de sus actuaciones, de resultar equivocadas. Asimismo, asumir la necesidad de entenderse como actores en el acceso de la justicia: la existencia del Derecho como ciencia (a la que considero también una vocación y un arte), tiene, una utilidad fundamental: pese a sus errores, vacíos y lagunas, como el mejor modelo 
generado por nuestra civilización para solucionar sus divergencias, y en suma, para modelar una sociedad mejor ${ }^{39}$. Para esto, y para finalizar este texto, se proponen tres temas básicos de debate: las Facultades deberían discutir: (1) un núcleo duro de creencias que sustente un modo de ilustrar el sistema normativo (2) un sistema normativo entendido en sus virtudes y defectos (3) la institucionalidad propiamente dicha, a lo que debería sumarse un 4) la administración de justicia como institución ser capaz de propiciar la solución de los problemas estructurales sociales que impiden el acceso a la solución de controversias. Solo así se puede construir una auténtica base que sea capaz de hacer una educación jurídica más real y, en consecuencia, un Derecho mejor.

\section{REFERENCIAS BIBLIOGRÁFICAS}

Almog, S. (2003). On Law and Utopia: Rules vs. Principles? - A Comment on Ramiro Avilés's Reply. Utopian Studies, 14, 143-148.

Ávila Lizán, L. (2008). Acceso a la justicia y emancipación social. In R. (. Ávila Santamaría, Neoconstitucionalismo y Sociedad (163-216). Quito, Ecuador: Ministerio de Justicia y Derechos Humanos.

Avilés, R. (2003). On Law and Utopia: A Reply to Shulamit Almog. Utopian Studies, 14(1), 133-142.

Binder, A. (2002). La fragmentación de la profesión judicial y la uniformidad de la carrera judicial. Santiago, Chile: CEJA.

Bobbio, N. (2001). Liberalismo y democracia. México D.F., México: Fondo de Cultura Económica.

Camps, V. (1998). Evolución y características de los derechos humanos. Los fundamentos de los derechos humanos desde la filosofía y el derecho. Madrid, España: Colectivo EDAI.

39 Al respecto, Bobbio (2001) es muy claro "Se puede hablar de estado de derecho en sentido profundo para distinguirlo del estado de derecho en sentido débil, que es el estado no despótico, es decir, no regido por los hombres sino por las leyes". 
Chase, L. E. (2002). La reforma del Estado. En J. Fernández Ruiz, Perspectivas del Derecho Administrativo en el Siglo XXI (151-162). México, D.F., México: UNAM.

Christie, G. (1987). The Recruitment of Law Faculty. Duke Law Journal, 306-316.

Ferrajoli, L. (1999). Derecho y Garantías, la Ley del más Débil. Madrid, España: Trotta.

Ferrajoli, L. (2013). Poderes salvajes: la crisis de la democracia constitucional. Madrid: Trotta.

Ferry, J. (1868). Lamentables etudes du droit. Le Temps. 3.

Kelsen, H. (1992). Introduction to the Problems of Legal Theory. (Litschewski Paulson, B. and Pau, S. Trans.) Oxford: Clarendon Press.

Medina, D. (2004). Teoría impura del derecho: la transformación de la cultura jurídica latinoamericana. Bogotá, Colombia: Legis.

Melin, G. and Janson, K. (2006). What skills and knowledge should a PhD have? Changing preconditions for $\mathrm{PhD}$ education and post doc work. In U. (. Teichler, The Formative Years of Scholars, Wenner-Gren International Studies (105-117). Londres: Portland Press.

O’Donnell, G. (2010). Revisitando el concepto de democracia delegativa. Casa del Tiempo, III(31), 1-8.

Pearce, R. G. (1998). Teaching Ethics Seriously: Legal Ethics as the Most Important Subject in Law School. Loyola University Chicago Law Journal, 29(4), 719-739.

Pollit, C., y Bouckaert, G. (2010). La reforma de la gestión púbica, un análisis comparado. (I. N. Pública, Ed.) Madrid, España: Instituto Nacional de Administración Pública.

Rivadeneyra, M. (1847). Los códigos españoles, concordados y anotados. Liber iudicium ..., Volumen 1. Madrid: Imprenta de la Publicidad. 
Savage, J. (2008). The Social Function of the Law Faculty: Demographics, Republican Reform, and Professional Training at the Paris Law Faculty, 1870-1914. History of Education Quarterly, 48(2), 221-243.

Schiltz, P. (1998). Legal Ethics in Decline: The Elite Law Firm, the Elite Law School, and the Moral Formation of the Novice Attorney. Minnsesota Law Reviwe(705), 746-787.

Shaffer, A. and Shaffer, R. (1960). The Law, Faculty Desegregation and Social Change. Phylon, 31(1), 38-47.

Simon, F., y Navas, M. (2003). Diagnóstico de la enseñanza legal de los Derechos Humanos en el Ecuador. Quito, Ecuador: Universidad San Francisco de Quito.

Thorseson, R. (1984). The Professor at Risk: Alcohol Abuse in Academe. The Journal of Higher Education, 55(1), 56-72.

Tremblay, R. (2016). Humankind's future: social and political Utopia or Idiocracy? Retrieved from OpEdNews: http://www.opednews.com/articles/Humankind-s-future-social-by-Roland-Michel-Trem-091101-248.html

University of California, Berkeley. (2014). Top Predictors of Graduate Student Well-Being. Universidad de Berkeley. Universidad de Berkeley.

Yudkowsky, E. (2007). Three major singularity schools. Retrieved diciembre 30, 2016, from Singularities: http://yudkowsky.net/singularity/schools 
Recibido: 26/12/2017

Aceptado: $24 / 05 / 2018$

Dr. Efrén Ernesto Guerrero Salgado: Docente Titular Principal PUCE Quito

Correo electrónico: matamur@gmail.com 